\title{
Endoscopic ablation is a cost-effective cancer preventative therapy in patients with Barrett's esophagus who have elevated genomic instability
}

Authors

Institutions
Ananya Das ${ }^{1}$, Keith M. Callenberg ${ }^{2,3}$, Mindi A. Styn ${ }^{2,3}$, Sara A. Jackson ${ }^{3}$

Arizona Center for Digestive Health, Gilbert, Arizona, USA

University of Pittsburgh Medical Center, Pittsburgh, Pennsylvania, USA

${ }^{3}$ Interpace Diagnostics Corporation (formerly RedPath Integrated Pathology), Pittsburgh, Pennsylvania, USA submitted:

28. October 2015

accepted after revision:

8. February 2016

\section{Bibliography}

DOI http://dx.doi.org/

10.1055/s-0042-103415

Published online: 15.4.2016

Endoscopy International Open

2016; 04: E549-E559

(c) Georg Thieme Verlag KG

Stuttgart · New York

E-ISSN 2196-9736

Corresponding author

\section{Ananya Das, MDF}

Arizona Center for Digestive Health

2680 South Valvista Drive,

Suite \#116

Gilbert, AZ 85295

USA

Fax: +1-412-224-6110

adas@azcdh.com
Background: The surveillance of patients with nondysplastic Barrett's esophagus (NDBE) has a high cost and is of limited effectiveness in preventing esophageal adenocarcinoma (EAC). Ablation for NDBE remains expensive and controversial. Biomarkers of genomic instability have shown promise in identifying patients with NDBE at high risk for progression to EAC. Here, we evaluate the cost-effectiveness of using such biomarkers to stratify patients with NDBE by risk for EAC and, subsequently, the cost-effectiveness of ablative therapy.

Methods: A Markov decision tree was used to evaluate four strategies in a hypothetical cohort of 50-year old patients with NDBE over their lifetime: strategy I, natural history without surveillance; strategy II, surveillance per current guidelines; strategy III, ablation for all patients; strategy IV, risk stratification with use of a biomarker panel to assess genomic instability (i.e., mutational load [ML]). Patients with no ML underwent

\section{Introduction}

\section{$\nabla$}

Over the last decade, significant advances have been made in our understanding of the natural history of Barrett's esophagus and the subsequent risk for progression to advanced neoplasia. In addition, the endoscopic ablative treatment of advanced neoplasia has improved markedly. However, the management of nondysplastic Barrett's esophagus (NDBE) continues to be based primarily on conservative endoscopic surveillance [1]. The assumptions that endoscopic surveillance can accurately detect progression to high grade dysplasia (HGD) and esophageal adenocarcinoma (EAC) and that early intervention makes a positive impact on survival have not yet been demonstrated in any large controlled prospective trials. Logistical, cost, and ethical issues severely limit the feasibility of such trials. However, a series of well-conducted economic analyses looking into minimal surveillance, patients with low ML underwent standard surveillance, and patients with high ML underwent ablation. The incremental cost-effectiveness ratio (ICER) and incremental net health benefit (INHB) were assessed.

Results: Strategy IV provided the best values for quality-adjusted life years (QALYs), ICER, and INHB in comparison with strategies II and III. Results were robust in sensitivity analysis. In a Monte Carlo analysis, the relative risk for the development of cancer in the patients managed with strategy IV was decreased. Critical determinants of strategy IV cost-effectiveness were the complete response rate, cost of ablation, and surveillance interval in patients with no ML.

Conclusion: The use of ML to stratify patients with NDBE by risk was the most cost-effective strategy for preventive EAC treatment. Targeting ablation toward patients with high ML presents an opportunity for a paradigm shift in the management of NDBE.

the most cost-effective management of NDBE have consistently demonstrated that expectant periodic endoscopic surveillance is costly and ineffective [2,3]. Our earlier work indicated that an aggressive strategy of endoscopic ablation in all patients with NDBE is generally not cost-effective and would be cost-effective only if targeted to a high risk subset of patients [4]. Therefore, additional management strategies, particularly ones that include risk stratification, need to be developed and evaluated.

Molecular biomarkers of genomic instability, including loss of heterozygosity (LOH) mutations near genes encoding tumor suppressor proteins (TP53 and CDKN2A), are associated with neoplastic progression in patients who have NDBE [5-7]. Panels of such biomarkers have the potential to provide a longer detection window and, subsequently, early intervention in patients with NDBE based on their risk for neoplastic progression. Un- 


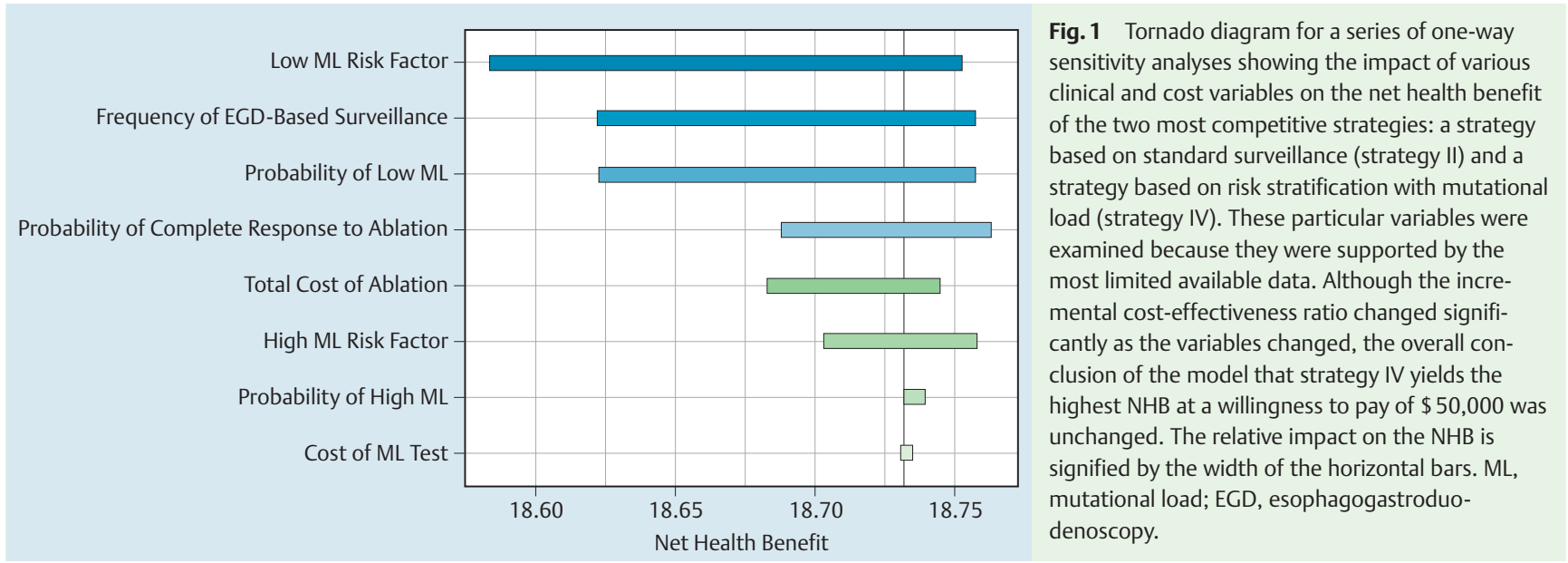

til fairly recently, this molecular testing was limited to academic and research efforts in which fresh tissue was used. However, testing can now be performed on formalin-fixed, paraffin-embedded tissue slides in a central laboratory, obviating the need for fresh tissue. These advancements have led to the development of a commercially available biomarker panel for assessing genomic instability that can be used in the clinical management of patients with Barrett's esophagus.

Mutational load (ML) is a summary measure of genomic instability across a panel of biomarkers often mutated in EAC [5,7]. ML assesses the presence and extent of genomic instability by measuring the number and clonality of $\mathrm{LOH}$ mutations in tissue with Barrett's esophagus - related histology. Mutations interrogated include LOH in 17p (TP53), 9p (CDKN2A), $1 \mathrm{p}$ (CMM1, L-myc), $3 \mathrm{p}$ (VHL, HoGG1), 5q (MCC, APC), 10q (PTEN, MXI1), 17q (NME1), 18q (DCC), 21q (TFF1, PSEN2), and 22q (NF2) genomic loci. ML also assesses the presence of microsatellite instability (MSI) at these loci. In cross-sectional studies, a small percentage of NDBE-derived histological tissue had ML similar to that of higher risk histological tissue (i.e., HGD or EAC), suggesting that ML may be predictive of impending, higher risk morphological changes $[5,7]$. Findings from a longitudinal study further demonstrate that patients who have NDBE that eventually progresses to HGD or EAC have elevated ML before the onset of histological progression [8]. Thus, ML assessment has the potential to improve patient management by providing a measure of genomic instability that could signal increased risk for future progression to EAC.

Herein, we update and build upon our previous analysis of the cost-effectiveness of Barrett's ablation strategies. We compare the current American College of Gastroenterology (ACG) guideline-recommended standard of care for patients who have NDBE with one in which ablation is indicated only for patients with NDBE who have a high risk for progression based on overall levels of genomic instability $[1,9]$. We report cost savings and incremental gains in quality-adjusted life years with the use of MLbased risk stratification and subsequent selective endoscopic ablative therapy for the preventative treatment of EAC.

\section{Methods}

$\nabla$

In this decision analysis, we considered a hypothetical cohort of white male patients with a mean age of 50 years in whom NDBE had recently been diagnosed during esophagogastroduodenoscopy (EGD) based on the ACG definition [10].

\section{Model}

We used commercially available decision analysis software (TreeAge Pro; TreeAge Software, Williamstown, Massachusetts, USA) to develop a hybrid model of a linear decision tree terminating in a Markov model to compare different strategies for the management of NDBE ( Supplementary Fig. 1) [11]. Various health and disease states (e.g., healthy, NDBE, Barrett's esophagus with low grade dysplasia (LGD), esophageal cancer, postoperative state, and finally death), each associated with a different set of costs and utilities ( $\checkmark$ Supplementary Fig. 2 ), were used to model the natural history of patients with Barrett's esophagus. At entry into the model, it was considered that all members of the cohort had already undergone the index EGD confirming the diagnosis of Barrett's esophagus without surface nodularity or masses and on biopsy would have only NDBE. At the end of each 1-year cycle of the model, they would be redistributed to different states depending on the estimated transitional probabilities among the various health states. Death was considered an absorbing state. The time horizon of the model was the lifetime of the cohort. The analysis was conducted according to the recommendations of the Panel on Cost-effectiveness in Health and Medicine for conducting and reporting a reference case analysis with a societal perspective [12].

\section{Strategies compared}

Four strategies were compared (Supplementary Methods):

Strategy I. "Natural history" of NDBE: No surveillance or interventions were used for NDBE with this strategy.

Strategy II. "Guideline-recommended surveillance" of NDBE: This strategy followed the ACG treatment guidelines for NDBE.

Strategy III. "Ablate all" patients with NDBE: Preventative endoscopic ablation therapy for NDBE was modeled primarily after a stepwise ablation procedure with the HALO Ablation System (Barrx Medical, Sunnyvale, California, USA).

Strategy IV. "ML-based risk stratification" according to levels of genomic instability: ML was assessed with BarreGen and PathFinderTG (Interpace Diagnostics, Pittsburgh, Pennsylvania, USA; 
formerly RedPath Integrated Pathology) in esophageal biopsy specimens from patients with NDBE, and preventative ablation was performed based on each patient's risk for progression to EAC.

\section{Clinical probabilities}

Clinical probabilities, including transitional probabilities and performance characteristics of endoscopy and biopsy in identifying different Barrett's esophagus - related health states, were derived from the published literature. Literature references for clinical probabilities are presented in the Supplementary Methods and Supplementary Table 1.

\section{Cost estimates and utilities}

Costs, not charges, were considered in this analysis, and a thirdparty payer's perspective was taken (Supplementary Methods and $\diamond$ Supplementary Table 2 ). Only direct costs were considered, and all costs were adjusted to 2013 U.S. dollars. Costs were estimated based on the national average reimbursement allowed for each coded procedure by the Centers for Medicare \& Medicaid Services (CMS) during the fiscal year 2013.

\section{Sensitivity analysis}

Model robustness was evaluated with sensitivity analysis based on important clinical probabilities and cost estimates. A secondorder Monte Carlo simulation was performed in the hypothetical cohort of 10,000 patients with NDBE for a probabilistic sensitivity analysis. This simulation recalculates a model multiple times, incorporating uncertainties into an analysis consistent with reallife situations [13]. Using tracker variables, we compared the number of patients in whom EAC developed with the different strategies.

\section{Outcomes compared and statistical methods}

The incremental cost-effectiveness ratio (ICER) and incremental net health benefit (INHB) were the primary outcomes compared among the four strategies. ICER is the difference in costs between strategies divided by the difference in outcome (life years) between strategies: for example, ICER $=$ (Cost Strategy I Cost Strategy II)/(Effectiveness Strategy I - Effectiveness Strategy II)]. The ICER is a measure of the added cost for each additional life year gained with a given strategy relative to another strategy.

The net health benefit (NHB) has been increasingly used in the economic evaluation of health care intervention as an alternative to the ICER. The NHB is a function of effectiveness (E), cost (C), and willingness to pay (WTP; i.e., the decision maker's threshold
ICER) according to the following formula: $\mathrm{NHB}=\mathrm{E}-\mathrm{C} / \mathrm{WTP})[14$, 15]. The NHB is the health effect of a treatment minus the benefit that one would have obtained by investing the resources spent on a marginally effective treatment. INHB) is the difference between two NHBs. INHB is emphasized here because it is often preferred to ICER as a measure of cost-effectiveness as it is a direct interpretation of the average health gained per patient who takes the different treatment adjusted for cost and WTP. Also, the INHB, as a monotonic function of both health and cost, tends to be easier to interpret; higher values are always better. Health policy makers would favor a strategy for which the INHB takes the greatest positive value in relation to values of WTP that seem reasonable with respect to known public policy.

To evaluate the results of the Monte Carlo analysis, relative risk (RR) with 95\% confidence intervals (CIs) and number needed to treat (NNT) were calculated. NNT was defined as the number of patients that needed to be treated per a selected strategy to prevent one case of esophageal cancer.

\section{Assumptions}

This model was based on several well-accepted, published assumptions used in decision analysis models for the management of Barrett's esophagus (Supplementary Methods).

\section{Results \\ $\nabla$}

\section{Baseline analysis}

In our baseline analysis, 50-year old subjects with NDBE who received no preventative intervention and followed the natural history of NDBE progression to HGD (strategy I) had an average of 17.567 quality-adjusted life years (QALYs) at a cost of $\$ 12,294$ per patient ( $\bullet$ Table 1 ). Compared with patients managed under strategy I, those managed with guideline-recommended endoscopic surveillance (strategy II) gained an additional 0.656 QALY, but at an incremental cost of $\$ 9068$ ( Table 1). Endoscopic ablation for all patients with NDBE (strategy III) had a slightly lower cost per patient than strategy II. Regardless, this cost remained incrementally higher than strategy I (\$7033) with only a small gain in QALYs (0.616) ( $\bullet$ Table 1). Compared with strategies II and III, risk stratification with ML (strategy IV) was the preferred strategy from a cost, QALY, and ICER perspective, with the highest yield of QALYs and lowest average cost per patient. Strategy IV remained the best strategy with the lowest cost and highest number of QALYs in a modified baseline analysis with the lowest MLbased risk stratification threshold (i.e.,"low ML”) as an indicator for ablation ( Table 1 ). In this modified analysis, any positive

Table 1 Results of baseline analysis comparing all management strategies for patients with nondysplastic Barrett's esophagus.

\begin{tabular}{|c|c|c|c|c|c|c|}
\hline \multirow{2}{*}{\multicolumn{2}{|c|}{ Strategy }} & Cost, $\$^{*}$ & QALYs & ICER (\$/QALY) & ICER (\$/QALY) & ICER (\$/QALY) \\
\hline & & & gained, y & vs. strategy I & vs. strategy II & vs. strategy III \\
\hline \multicolumn{2}{|l|}{ I. Natural history } & 12,924 & 17.567 & - & 13,823 & 11,417 \\
\hline \multicolumn{2}{|c|}{ II. Guideline-recommended surveillance } & 21,992 & 18.223 & 13,823 & - & 2031 \\
\hline \multicolumn{2}{|l|}{ III. Ablate all } & 19,957 & 18.183 & 11,417 & 2031 & - \\
\hline \multirow[t]{2}{*}{$\begin{array}{l}\text { IV. ML-based risk } \\
\text { stratification }\end{array}$} & $\begin{array}{l}\text { Patients with "high ML" } \\
\text { undergo ablation. }\end{array}$ & 17,234 & 19.081 & 2847 & -5545 & -3032 \\
\hline & $\begin{array}{l}\text { Patients with "high ML" and } \\
\text { "low ML" undergo ablation. }\end{array}$ & 16,461 & 18.763 & 2957 & $-10,243$ & $-6,028$ \\
\hline
\end{tabular}

QALYs, quality-adjusted life years; ICER, incremental cost-effectiveness ratio; ML, mutational load.

* Direct costs of care per patient (listed in Supplemental Table 2) from a third-party perspective accrued over the lifetime of patients as they transition from one health state to another based on the different transitional probabilities in yearly cycles, as modeled in the decision tree. 
level of ML (i.e., both "low ML" and "high ML") was considered an indication for ablation (patients with "no ML" were excluded).

\section{Monte Carlo analysis}

In the Monte Carlo simulation, a total of 831 esophageal cancers developed in the natural course of NDBE (strategy I) over a calculated period of 174,853 person-years, for an average risk of $0.47 \%$ per person-year. The numbers of esophageal cancers that were diagnosed under each strategy during the lifetime of this cohort were estimated at $831,819,596$, and 402 for strategies I, II, III and IV, respectively. Thus, compared with a strategy of no preventative intervention (strategy I), the RR for the development of cancer with ML-based risk stratification (strategy IV) remained low $(0.48 \%, 95 \%$ CI $0.43-0.54)$; the NNT for preventing cancer with this strategy was only 23 (95\%CI 20-28). Similarly, compared with guideline-recommended endoscopic surveillance (strategy II), the RR for the development of esophageal cancer with MLbased risk stratification (strategy IV) was low $(0.49 \%, 95 \% \mathrm{CI}$ $0.44-0.55)$, with an NNT of only 24 (95\%CI $21-28$ ).

- Supplemental Fig. 2 shows the proportions of iterations in the Monte Carlo analysis (y-axis) that are acceptable as cost-effective for each strategy against increasing WTP (x-axis). For all levels of WTP, a strategy of ML-based risk stratification (strategy IV) was the most cost-effective strategy in terms of incremental NHB (INHB), particularly when compared against the currently practiced guideline-recommended strategy of endoscopic surveillance (strategy II).

\section{Sensitivity analysis}

When a one-way sensitivity analysis was performed by varying important clinical probability and cost estimates, the model was sensitive to several clinically important model parameters. - Fig. 1 is a Tornado diagram of a series of one-way sensitivity analyses for NHB showing the different clinical and cost variables that impacted the NHB values of the two most competing strategies (i.e., guideline-recommended surveillance [strategy II] and ML-based risk stratification [strategy IV]). These particular variables were examined because they were supported by the most limited data. It is important to note that although the outcome values in dollar amounts changed as imputed values changed, the overall conclusion of the model with respect to NHB was unchanged; strategy IV yielded the highest NHB across a wide range of WTPs including a WTP of $\$ 50,000$, which is routinely considered the threshold for an intervention to be cost-effective ( $\bullet$ Supplementary Fig.3). Other important clinical parameters, such as the rate of complications with ablative therapy, frequency of endoscopic surveillance after complete ablation, annual probability of progression of HGD to cancer, quality of life after esophagectomy, and mortality rate after esophagectomy, did not significantly impact the conclusion of the baseline analysis (data not shown).

\section{Discussion}

$\nabla$

In this cost-effectiveness analysis, we show that among competing strategies for managing patients with NDBE, risk stratification according to levels of genomic instability, with an ML-based approach, is superior to other strategies of patient management. Most importantly, this approach dominates the ACG [1,9] and other [16] guideline-recommended strategies for endoscopic surveillance. ML-based risk stratification not only yields the highest number of QALYs for patients but also costs less, resulting in comparatively lower costs per QALY gained (ICER). Moreover, it yields the highest INHB to patients, which remains robust when important cost and clinical variables are varied in sensitivity analysis. We used a modified version of a previously published decision analysis model, the internal validity of which was corroborated by the estimated lifetime risk of approximately $0.5 \%$ per person-year for the development of EAC in the natural history arm. Such risk is supported by population-based studies of the natural history of actual patients with Barrett's esophagus demonstrating an incidence of malignancy of approximately $0.5 \%$ per year

Recent prospective studies have shown the safety, tolerability, and efficacy of endoscopic ablative therapy specifically in patients with NDBE [17-19]. With well-designed studies showing high rates of complete elimination of intestinal metaplasia and eradication of pre-ablation oncogenetic abnormalities following ablation, concerns about the recurrence of metaplasia and the durability of post-ablation neo-squamous epithelium are decreasing [20,21]. Recently, in another economic analysis, we studied the cost-effectiveness of a strategy based on preventative endoscopic ablative therapy in all patients with NDBE [4]. Although the strategy yielded a higher number of QALYs compared with a guideline-recommended strategy of endoscopic surveillance, the cost-effectiveness of such a strategy was borderline, given the high cost of endoscopic ablation. Thus, ablation for all patients with NDBE could not be recommended for clinical practice from a health economics perspective. Of note, this economic analysis clearly called for a risk stratification strategy to identify and selectively perform ablation only in patients with NDBE who are at high risk for progression to advanced neoplasia. Consistently, the management strategy that included ML-based risk stratification of patients with NDBE, which is examined herein, was more cost-effective than ablation for all patients with NDBE. Several biomarkers, such as DNA content abnormality (aneuploidy, tetraploidy), abnormalities at tumor suppressor gene loci (17 $\mathrm{p}$ and $9 \mathrm{p} \mathrm{LOH}$ ), epigenetic changes (CDKN2A methylation), cell cycle markers (cyclin D1 expression), and proliferation markers, have been associated with progression from NDBE to EAC [5-7]. In a landmark longitudinal study, Galipeau et al. [6] showed that a panel of abnormalities including $17 \mathrm{p} \mathrm{LOH}$, DNA content irregularities (tetraploidy and aneuploidy), and $9 \mathrm{p} \mathrm{LOH}$ was the best predictor of esophageal cancer in comparison with individual biomarkers examined (RR 38.7, 95\%CI 10.8-138.5, P=0.001). Patients with no baseline abnormality had a 10 -year cumulative esophageal cancer incidence of $12 \%$, whereas patients with three abnormalities (17 p LOH, DNA aneuploidy, and 9 p LOH) had a 10year cancer incidence of at least $79.1 \%$ [6].

Additional studies have shown that risk for progression to EAC increases as the clonal expansion of cells with such mutations increases [22-24]. Khara et al. recently reported the presence and extent of genomic instability by using ML in patients with NDBE and other, higher histological grades of disease [7]. ML incorporated the presence and clonality of LOH mutations next to TP53 and CDKN2A, as well as eight additional genomic loci in proximity to other tumor suppressor genes. MSI mutation at these loci was also included in ML. Although some LOH and MSI damage was detected in NDBE, much more was accumulated in advanced stages of Barrett's histology. A "high ML" level was present in up to $95 \%$ and $96 \%$ of patients with HGD and EAC, respectively, whereas only $8 \%$ of patients with NDBE had a similar "high ML" level [7]. In another, related longitudinal study of 69 patients 
with Barrett's esophagus, Eluri et al. showed that patients with Barrett's esophagus who had no or low levels of dysplasia but "high ML" levels were more likely to progress to HGD or EAC at a mean follow-up period of 4 years (adjusted odds ratio [OR] 166, $P$ $<0.0001$ ) [8].

A concern regarding the risk stratification of patients with NDBE based on biomarker panels has been the perceived high cost of biomarker testing, which led us to perform this economic analysis. Two previous decision analyses incorporated biomarkerbased risk stratification strategies for the management of patients with NDBE $[25,26]$. Both studies considered hypothetical biomarkers and were limited by significant assumptions regarding the performance characteristics and cost of these hypothetical tests. The earlier study did not consider endoscopic ablative therapy at all because it was not clinically available at the time of analysis [26]. The recent study by Gordon et al. focused on the ability of biomarkers to modify NDBE surveillance intervals but did not incorporate endoscopic ablative therapy as a primary intervention [25]. In our earlier work, we have shown that blanket endoscopic ablative therapy for NDBE is only borderline cost-effective [4]. We have now furthered such analysis to show that limiting preventative endoscopic ablative therapy to those patients with NDBE at high risk for progression to HGD or EAC makes ablation convincingly cost-effective compared with current guideline-recommended endoscopic surveillance management.

Although our model shows promising cost-effectiveness for preventative ablation when it is limited to high risk patients with the use of biomarkers, it does have limitations. We did not account for any indirect costs and considered only stepwise radiofrequency ablation as the method of endoscopic ablation, although other ablation techniques are available. For patients with "low ML," the endoscopic surveillance intervals were arbitrarily decided based on expert opinion, given the lack of robust long-term data on the risk for progression in these patients. However, sensitivity analyses showed that even ablation in all patients at lower risk for progression (i.e., those with "low ML") was cost-effective and improved QALYs. The model also assumed that all specimens for ML analysis represented the worst Barrett's-related histology present in the patient, which is a strong assumption, given the sampling variability associated with biopsy in these patients.

In summary, this economic analysis examined a strategy of using ML-based risk stratification with a commercially available panel of DNA markers to assess overall genomic instability in patients with NDBE. When endoscopic ablative therapy was selectively applied in patients at high risk for progression, the management of patients with NDBE was superior to the current guideline-recommended management strategy of conservative surveillance. Larger longitudinal studies of the use of biomarkers, such as ML, in combination with established clinical and endoscopic predictors to enhance risk stratification are urgently needed to convince clinicians and policy makers to change the current costly and ineffective practice of endoscopic surveillance.

\section{Supplemental methods}

\section{$\nabla$}

\section{Strategies compared}

Four strategies were compared:

Strategy I. "Natural history" of nondysplastic Barrett's esophagus (NDBE): No surveillance or interventions were used for NDBE with this strategy. Patients underwent esophagogastroduodeno- scopy (EGD) only if a symptom suggestive of cancer, such as dysphagia or weight loss, developed. If cancer was discovered, patients with unresectable disease received palliative care (e.g., endoscopic therapy, chemoradiotherapy, hospice care). For those with resectable disease, the model accounted for surgery-related morbidity and mortality; it also accounted for the risk for recurrence. In addition to rates of mortality related to esophageal cancer, the U.S.life table mortality rates were incorporated into the model to account for age- and gender-specific annual mortality from all other causes.

Strategy II. "Guideline-recommended surveillance" of NDBE: This strategy followed the American College of Gastroenterology (ACG) treatment guidelines for NDBE. Patients with NDBE underwent surveillance every 3 years. If low grade dysplasia (LGD) was discovered, the frequency of surveillance was increased to annual until no dysplasia could be detected. If high grade dysplasia (HGD) was discovered, patients underwent ablation as detailed in strategy III. Patients with focal/nodular HGD underwent repeat EGD and endoscopic mucosal resection followed by ablation. Esophagectomy was considered for patients with esophageal adenocarcinoma (EAC) or persistent, diffuse, or multifocal HGD. When indicated, endoscopic therapy was performed for patients with nodular HGD or EAC. The possibility of misdiagnosis of histologic specimens, a common problem in Barrett's esophagus, was included in the model based on published false-positive and falsenegative rates. Surveillance continued to age 80 .

Strategy III. "Ablate all" patients with NDBE: Preventative endoscopic ablation therapy for NDBE was modeled primarily after a stepwise ablation procedure with the HALO Ablation System (Barrx Medical, Sunnyvale, California, USA). All patients who had NDBE underwent preventative endoscopic ablation up to three times. Each time, patients incurred a risk for complications. Patients with perforations as a result of ablation were assumed to undergo esophagectomy. As noted for strategy I, the model accounted for surgery-related morbidity and mortality. Patients who had esophageal strictures as a result of ablation were assumed to undergo dilation; risks associated with dilation were included in the model. When ablation was successful, patients underwent surveillance annually for 3 years and then every 10 years in baseline analysis, with a range of 3 to every 10 years for sensitivity analysis. When ablation was incomplete, patients underwent surveillance as in strategy II and were considered to have the same risk for progression as at baseline.

Strategy IV. "Mutational load (ML)- based risk stratification" according to levels of genomic instability: ML was assessed with BarreGen and PathFinderTG (Interpace Diagnostics, Sunnyvale, California, USA; formerly RedPath Integrated Pathology) in esophageal biopsy specimens from patients with NDBE, and preventative ablation was performed based on each patient's risk for progression to EAC. Patients with NDBE and minimal risk for progression to HGD or EAC (i.e., those with "no ML") were followed as in strategy II, except that they underwent endoscopic surveillance every 10 years. Patients with a low risk for progression to HGD or EAC (i.e., those with "low ML") were followed as in strategy II, although the model incorporated a second decision node to examine the possibility of managing these patients with an aggressive strategy in which they underwent endoscopic ablative therapy. Patients with a high risk for progression to HGD or EAC (i.e., those with "high ML") underwent ablation as in strategy III. All patients with "high ML" underwent endoscopic surveillance once a year indefinitely, even if complete eradication of intestinal metaplasia was achieved. 


\section{Clinical probabilities}

The MEDLINE database and abstracts from major gastroenterology meetings were searched for all relevant articles published from 1996 to 2015 by using the terms Barrett's esophagus, esophageal cancer, gastroesophageal reflux disease, economic analysis, radiofrequency ablation, and photodynamic therapy. Also, the references of selected publications were searched manually. When specific published information was not available, expert opinion was obtained by consensus. Supplementary Table 2 lists the clinical probabilities imputed into the model.

Literature exists on the prevalence of ML in patients with NDBE, HGD, and EAC, as well as on the relative risk for progression from NDBE to EAC based on levels of genomic instability. Prevalence data for ML were primarily obtained from a recent study that assessed ML in 427 patients with NDBE; in this study, 30\% had "no ML," $62 \%$ had "low ML," and $8 \%$ had "high ML" [1]. The ML-based risk for progression from NDBE to HGD was obtained from a review of the cumulative published literature citing genomic instability as a risk factor in progression of NDBE to EAC. To assess genomic instability, ML measures both loss of heterozygosity (LOH) and microsatellite instability (MSI) mutations at $9 \mathrm{p}$ (CDKN2A) and $17 \mathrm{p}$ (TP53), as well as at 8 additional genomic loci: 1 p (CMM1, L-myc), 3 p (VHL, HoGG1), 5q (MCC, APC), 10q (PTEN, MXI1), 17q (NME1), 18q (DCC), 21q (TFF1, PSEN2) and 22q (NF2) $[27,28]$. In a longitudinal study, 243 patients with NDBE were evaluated at baseline for genomic instability by examining DNA content abnormalities (tetraploidy, aneuploidy) and TP53 (17p) and CDKN2A (9p) alterations (methylation, point mutations, and LOH). At 10 years, all abnormalities, except CDKN2A point mutation and methylation, significantly contributed to risk for progression to HGD and EAC, with relative risk by univariate analysis ranging from 2.6 (for $9 p \mathrm{LOH}$ ) to 10.6 (for $17 \mathrm{p} \mathrm{LOH}$ ) [3]. A panel of abnormalities associated with genomic instability, including 17 p LOH, DNA content irregularity, and 9p LOH, was the best predictor of esophageal cancer, with a relative risk of 38.7 [29]. Additional studies have shown that risk for progression to EAC also increases with the increased clonal expansion of cells that have such cumulative DNA damage 30]. ML assesses both the presence and extent (i.e., clonality) of such mutations in Barrett's tissue $[27,28]$. Consistently, in a recent longitudinal study of ML, the presence of "high ML" was highly predictive of progression to HGD or EAC, with an adjusted odds ratio of 166 for progression at a mean follow-up of 4 years $(P<0.0001)$ [31].

Numbers extrapolated from the above studies were used in our cost analysis study to evaluate the impact of biomarkers of genomic instability assessed by ML-based risk stratification methods. In our baseline analysis for calculating annual rate of progression based on ML-associated risk, we used a conservative baseline risk ratio of 10 (range $0-25$ ) for patients with low risk (i.e., those with "low ML") and of 25 (range 5-50) for patients with high risk (i.e., those with "high ML") for progression of NDBE to HGD or EAC. All risk was relative to that of patients with minimal risk for progression (i.e., those with "no ML"). A wide range of risks was examined in sensitivity analysis.

\section{Cost estimates and utilities}

Costs, not charges, were considered in this analysis, and a thirdparty payer's perspective was taken ( Supplementary Table 3 ). Only direct costs were considered, and all costs were adjusted to 2013 U.S.dollars. Costs were estimated based on the national average reimbursement allowed for each coded procedure by the Centers for Medicare \& Medicaid Services (CMS) during the fiscal year 2013. Inpatient medical, surgical, and diagnostic services were assigned CPT (current procedural terminology) or DRG (diagnosis-related group) codes to identify the health care resource utilization. Outpatient data were based on ambulatory payment classification and CPT ( $\bullet$ Supplementary Table 3 ). For sensitivity analysis, the range of the cost estimates was obtained from published information [32-37].

For baseline analysis, the cost of ML testing was estimated at $\$ 3100$; this figure was extrapolated from CMS reimbursement data for a similar assay related to integrated molecular pathology testing of pancreatic cystic neoplasm. The total cost of the endoscopic ablative therapy included the total cost of all procedures (performed up to 3 times) including professional fees and facility fees, which were assumed to be accrued in the first year of the model. The costs of ablation-related complications were accounted for separately.

Quality-adjusted life years (QALYs), which were the measure of effectiveness in this model, were estimated by adjusting the life expectancy of each health state by a weight or utility reflecting patient preferences for that health state. Utility values were obtained from published information ( $\bullet$ Supplementary Table 3 ).

\section{Assumptions}

The model and assumptions used were based on well-accepted and published decision analysis models for the management of Barrett's esophagus [6-11]. All patients with NDBE were considered potential candidates for surgical esophagectomy, if needed, and in the baseline analysis, the postoperative complication rates for esophagectomy were modeled by using data from high-volume academic centers. Any benefit in terms of reduced risk for cancer was presumed to be accrued only in the case of complete ablation of specialized columnar epithelium; patients with partial ablation had no reduction in their risk for cancer although they incurred the costs associated with endoscopic ablative therapy. Also, as previously mentioned, even patients with complete ablation continued to accrue the cost of periodic endoscopic surveillance.

We used conservative estimates in modeling the strategy based on endoscopic ablation because of the dearth of published data on Barrett's ablation with the stepwise ablation procedure. For example, although the published article on the ablation of Barrett's esophagus with the stepwise procedure reported a rate of complete ablation of $70 \%$ at 1 year and of $98 \%$ at 2.5 years, and no serious adverse event in 100 patients, in our baseline scenario we used a much more conservative estimate of $50 \%$ as a complete response rate and did incorporate an overall complication rate of $10 \%$ [38]. These estimates of complete response in Barrett's esophagus and safety with ablative therapy have since been confirmed in other trials of ablation in patients who have Barrett's esophagus with and without dysplasia [39-45]. In other assumptions, only direct costs were considered. In our experience, patients undergoing the stepwise ablation procedure for Barrett's esophagus on an outpatient basis tolerate the procedure quite well, and no forms of short-term disutility related to uncomplicated endoscopic ablation were considered in this model. Because the patients being managed according to each strategy would be receiving acid-suppressive therapy for gastroesophageal reflux disease (GERD), the cost of such treatment was not considered in the model. For the ML-based risk stratification strategy, we assumed that biopsy specimens were of sufficient quantity and quality for ML processing. 
Competing interests: A.D. receives research funding from Interpace Diagnostics (formerly RedPath). S.A.J., M.A.S., and K.M.C. are full-time employees of Interpace Diagnostics.

\section{References}

1 Spechler SJ, Sharma P, Souza RF et al. American Gastroenterological Association medical position statement on the management of Barrett's esophagus. Gastroenterology 2011; 140: 1084-1091

2 Inadomi J, Madanick R, Somsouk $M$ et al. Radiofrequency ablation is more cost-effective than endoscopic surveillance or esophagectomy among patients with Barrett's esophagus and low-grade dysplasia. Gastroenterology 2007; 132: A53

3 Inadomi JM, Sampliner R, Lagergren J et al. Screening and surveillance for Barrett esophagus in high-risk groups: a cost-utility analysis. Ann Intern Med 2003; 138: 176-186

4 Das A, Wells C, Kim HJ et al. An economic analysis of endoscopic ablative therapy for management of nondysplastic Barrett's esophagus. Endoscopy 2009; 41: 400-408

5 Ellsworth E, Jackson SA, Thakkar SJ et al. Correlation of the presence and extent of loss of heterozygosity mutations with histological classifications of Barrett's esophagus. BMC Gastroenterol 2012; 12: 181

6 Galipeau PC, Li X, Blount PL et al. NSAIDs modulate CDKN2A, TP53, and DNA content risk for progression to esophageal adenocarcinoma. PLoS Med 2007; 4: e67

7 Khara HS, Jackson SA, Nair S et al. Assessment of mutational load in biopsy tissue provides additional information about genomic instability to histological classifications of Barrett's esophagus. J Gastrointest Cancer 2014; 45: 137-145

8 Eluri S, Brugge WR, Daglilar ES et al. The presence of genetic mutations at key loci predicts progression to esophageal adenocarcinoma in Barrett's esophagus. Am J Gastroenterol 2015; 110: 828-834

9 Spechler SJ, Sharma P, Souza RF et al. American Gastroenterological Association technical review on the management of Barrett's esophagus. Gastroenterology 2011; 140: e18 - e52; quiz e13

10 Sampliner RE. Updated guidelines for the diagnosis, surveillance, and therapy of Barrett's esophagus. Am J Gastroenterol 2002; 97: 18881895

11 Sonnenberg FA, Beck JR. Markov models in medical decision making: a practical guide. Med Decis Making 1993; 13: 322-338

12 Weinstein MC, Siegel JE, Gold MR et al. Recommendations of the Panel on Cost-effectiveness in Health and Medicine. JAMA 1996; 276: $1253-1258$

13 Doubilet P, Begg CB, Weinstein MC et al. Probabilistic sensitivity analysis using Monte Carlo simulation. A practical approach. Med Decis Making 1985; 5: $157-177$

14 Heitjan DF. Fieller's method and net health benefits. Health Econ 2000; 9: $327-335$

15 Stinnett A, Mullahy J. Net health benefits: a new framework for the analysis of uncertainty in cost-effectiveness analysis. Med Decis Making 1998; 18: 68-S80

16 Fitzgerald $R C$, di Pietro $M$, Ragunath $K$ et al. British Society of Gastroenterology guidelines on the diagnosis and management of Barrett's oesophagus. Gut 2014; 63: 7-42

17 Fleischer DE, Overholt BF, Sharma VK et al. Endoscopic radiofrequency ablation for Barrett's esophagus: 5-year outcomes from a prospective multicenter trial. Endoscopy 2010; 42: $781-789$

18 Sharma VK, Wang KK, Overholt BF et al. Balloon-based, circumferential, endoscopic radiofrequency ablation of Barrett's esophagus: 1-year follow-up of 100 patients. Gastrointest Endosc 2007; 65: 185-195

19 Roorda AK, Marcus SN, Triadafilopoulos G. Early experience with radiofrequency energy ablation therapy for Barrett's esophagus with and without dysplasia. Dis Esophagus 2007; 20: 516-522

20 Phoa KN, van Vilsteren FG, Weusten BL et al. Radiofrequency ablation vs endoscopic surveillance for patients with Barrett esophagus and lowgrade dysplasia: a randomized clinical trial. JAMA 2014; 311: 12091217

21 Bulsiewicz WJ, Kim HP, Dellon ES et al. Safety and efficacy of endoscopic mucosal therapy with radiofrequency ablation for patients with neoplastic Barrett's esophagus. Clin Gastroenterol Hepatol 2013; 11: 636-642

22 Maley CC. Multistage carcinogenesis in Barrett's esophagus. Cancer Lett 2007; 245: $22-32$
23 Maley CC, Galipeau PC, Finley JC et al. Genetic clonal diversity predicts progression to esophageal adenocarcinoma. Nat Genet 2006; 38: 468 473

24 Maley CC, Galipeau PC, Li X et al. The combination of genetic instability and clonal expansion predicts progression to esophageal adenocarcinoma. Cancer Res 2004; 64: 7629-7633

25 Gordon LG, Mayne GC, Hirst NG et al. Cost-effectiveness of endoscopic surveillance of non-dysplastic Barrett's esophagus. Gastrointest Endosc 2014; 79: $242-256$

26 Rubenstein JH, Vakil N, Inadomi JM. The cost-effectiveness of biomarkers for predicting the development of oesophageal adenocarcinoma. Aliment Pharmacol Ther 2005; 22: 135-146

\section{Supplemental References}

27 Khara HS, Jackson SA, Nair S et al. Assessment of mutational load in biopsy tissue provides additional information about genomic instability to histological classifications of Barrett's esophagus. J Gastrointest Cancer 2014; 45: 137 - 145

28 Ellsworth E,Jackson SA, Thakkar SJ et al. Correlation of the presence and extent of loss of heterozygosity mutations with histological classifications of Barrett's esophagus. BMC Gastroenterol 2012; 12: 181

29 Galipeau PC, Li X, Blount PL et al. NSAIDs modulate CDKN2A, TP53, and DNA content risk for progression to esophageal adenocarcinoma. PLoS Med 2007; 4: e67

30 Maley CC, Galipeau PC, Li X et al. The combination of genetic instability and clonal expansion predicts progression to esophageal adenocarcinoma. Cancer Res 2004; 64: 7629-7633

31 Eluri S, Brugge WR, Daglilar ES et al. The presence of genetic mutations at key loci predicts progression to esophageal adenocarcinoma in Barrett's esophagus. Am J Gastroenterol 2015; 110: 828 -834

32 Gerson LB, Groeneveld PW, Triadafilopoulos G. Cost-effectiveness model of endoscopic screening and surveillance in patients with gastroesophageal reflux disease. Clin Gastroenterol Hepatol 2004; 2: 868 879

33 Hur C, Nishioka NS, Gazelle GS. Cost-effectiveness of aspirin chemoprevention for Barrett's esophagus. J Natl Cancer Inst 2004; 96: 316-325

34 Inadomi JM, Sampliner R, Lagergren J et al. Screening and surveillance for Barrett esophagus in high-risk groups: a cost-utility analysis. Ann Intern Med 2003; 138: 176-186

35 Shaheen NJ, Inadomi JM, Overholt BF et al. What is the best management strategy for high grade dysplasia in Barrett's oesophagus? A cost effectiveness analysis Gut 2004; 53: 1736 - 1744

36 Sonnenberg A, Fennerty MB. Medical decision analysis of chemoprevention against esophageal adenocarcinoma. Gastroenterology 2003; 124: $1758-1766$

37 Vij R, Triadafilopoulos G, Owens DK et al. Cost-effectiveness of photodynamic therapy for high-grade dysplasia in Barrett's esophagus. Gastrointest Endosc 2004; 60: 739-756

38 Sharma VK, Wang KK, Overholt BF et al. Balloon-based, circumferential, endoscopic radiofrequency ablation of Barrett's esophagus: 1-year follow-up of 100 patients. Gastrointest Endosc 2007; 65: 185 - 195

39 Fleischer DE, Overholt BF, Sharma VK et al. Endoscopic ablation of Barrett's esophagus: a multicenter study with 2.5 -year follow-up. Gastrointest Endosc 2008; 68: 867-876

40 Ganz RA, Overholt BF, Sharma VK et al. Circumferential ablation of Barrett's esophagus that contains high-grade dysplasia: a U.S. Multicenter Registry. Gastrointest Endosc 2008; 68: 35-40

41 Gondrie JJ, Pouw RE, Sondermeijer CM et al. Stepwise circumferential and focal ablation of Barrett's esophagus with high-grade dysplasia: results of the first prospective series of 11 patients. Endoscopy 2008; 40: $359-369$

42 Gondrie JJ, Pouw RE, Sondermeijer CM et al. Effective treatment of early Barrett's neoplasia with stepwise circumferential and focal ablation using the HALO system. Endoscopy 2008; 40: 370-379

43 Shaheen $N$, Sharma $P$, Overholt B et al. A randomized, multicenter, sham-controlled trial of radiofrequency ablation (RFA) for subjects with Barrett's esophagus (BE) containing dysplasia: interim results of the AIM Dysplasia Trial. Gastroenterology 2008; 134: A37

44 Sharma VK, Jae Kim H, Das A et al. Circumferential and focal ablation of Barrett's esophagus containing dysplasia. Am J Gastroenterol 2009; 104: $310-317$ 
45 Sharma VK, Kim HJ, Das A et al. A prospective pilot trial of ablation of Barrett's esophagus with low-grade dysplasia using stepwise circumferential and focal ablation (HALO system). Endoscopy 2008; 40: $380-387$

46 Schnell TG, Sontag SJ, Cheifec G et al. Long-term nonsurgical management of Barrett's esophagus with high-grade dysplasia. Gastroenterology 2001; 120: 1607-1619

47 Hameeteman W, Tytgat GN, Houthoff HJ et al. Barrett's esophagus: development of dysplasia and adenocarcinoma. Gastroenterology 1989; 96: $1249-1256$

48 Weston AP, Sharma P, Topalovski M et al. Long-term follow-up of Barrett's high-grade dysplasia. Am J Gastroenterol 2000; 95: 1888 - 1893

49 Sharma P, Morales TG, Bhattacharyya A et al. Dysplasia in short-segment Barrett's esophagus: a prospective 3-year follow-up. Am J Gastroenterol 1997; 92: 2012 - 2016

50 Weston AP, Badr AS, Hassanein RS. Prospective multivariate analysis of clinical, endoscopic, and histological factors predictive of the development of Barrett's multifocal high-grade dysplasia or adenocarcinoma. Am J Gastroenterol 1999; 94: 3413-3419

51 Cameron AJ, Ott BJ, Payne WS. The incidence of adenocarcinoma in columnar-lined (Barrett's) esophagus. N Engl J Med 1985; 313: 857-859

52 Drewitz DJ, Sampliner RE, Garewal HS. The incidence of adenocarcinoma in Barrett's esophagus: a prospective study of 170 patients followed 4.8 years. Am J Gastroenterol 1997; 92: 212 - 215

53 O'Connor JB, Falk GW, Richter JE. The incidence of adenocarcinoma and dysplasia in Barrett's esophagus: report on the Cleveland Clinic Barrett's Esophagus Registry. Am J Gastroenterol 1999; 94: 2037-2042

54 Shaheen NJ, Crosby MA, Bozymski EM et al. Is there publication bias in the reporting of cancer risk in Barrett's esophagus? Gastroenterology 2000; 119: $333-338$

55 Spechler SJ, Lee E, Ahnen D et al. Long-term outcome of medical and surgical therapies for gastroesophageal reflux disease: follow-up of a randomized controlled trial. JAMA 2001; 285: 2331-2338

$56 \mathrm{Katz} D$, Rothstein $R$, Schned $A$ et al. The development of dysplasia and adenocarcinoma during endoscopic surveillance of Barrett's esophagus. Am J Gastroenterol 1998; 93: 536-541

57 Spechler SJ, Robbins AH, Rubins HB et al. Adenocarcinoma and Barrett's esophagus. An overrated risk? Gastroenterology 1984; 87: 927-933

58 Weston AP, Badr AS, Hassanein RS. Prospective multivariate analysis of factors predictive of complete regression of Barrett's esophagus. Am J Gastroenterol 1999; 94: 3420-3426

59 Winters CJr, Spurling TJ, Chobanian SJ et al. Barrett's esophagus. A prevalent, occult complication of gastroesophageal reflux disease. Gastroenterology 1987; 92: 118-124

60 Corley DA, Levin TR, Habel LA et al. Surveillance and survival in Barrett's adenocarcinomas: a population-based study. Gastroenterology 2002; 122: $633-640$

61 Menke-Pluymers MB, Schoute NW, Mulder AH et al. Outcome of surgical treatment of adenocarcinoma in Barrett's oesophagus. Gut 1992; 33 : $1454-1458$

62 Pera M, Trastek VF, Carpenter HA et al. Barrett's esophagus with highgrade dysplasia: an indication for esophagectomy? Ann Thorac Surg 1992; 54: 199-204

63 Peters JH, Clark GW, Ireland AP et al. Outcome of adenocarcinoma arising in Barrett's esophagus in endoscopically surveyed and nonsurveyed patients. J Thorac Cardiovasc Surg 1994; 108: 813-821; discussion $821-822$

64 Streitz JMJr, Andrews CWJr, Ellis FHJr. Endoscopic surveillance of Barrett's esophagus. Does it help? J Thorac Cardiovasc Surg 1993; 105: 383 - 387; discussion 387-388

65 Overholt BF, Panjehpour M, Halberg DL. Photodynamic therapy for Barrett's esophagus with dysplasia and/or early stage carcinoma: longterm results. Gastrointest Endosc 2003; 58: 183-188
66 Sharma P, Wani S, Weston AP et al. A randomised controlled trial of ablation of Barrett's oesophagus with multipolar electrocoagulation versus argon plasma coagulation in combination with acid suppression: long term results. Gut 2006; 55: $1233-1239$

67 Bousamra M2nd, Haasler GB, Parviz M. A decade of experience with transthoracic and transhiatal esophagectomy. Am J Surg 2002; 183: $162-167$

68 Lerut T, Coosemans W, Van Raemdonck D et al. Surgical treatment of Barrett's carcinoma. Correlations between morphologic findings and prognosis. J Thorac Cardiovasc Surg 1994; 107: 1059-1065; discussion $1065-1056$

69 McLarty AJ, Deschamps C, Trastek VF et al. Esophageal resection for cancer of the esophagus: long-term function and quality of life. Ann Thorac Surg 1997; 63: $1568-1572$

70 Patti MG, Corvera CU, Glasgow RE et al. A hospital's annual rate of esophagectomy influences the operative mortality rate. J Gastrointest Surg 1998; 2: 186-192

71 Zaninotto G, Parenti AR, Ruol A et al. Oesophageal resection for highgrade dysplasia in Barrett's oesophagus. Br J Surg 2000; 87: 1102 1105

72 Arrowsmith JB, Gerstman BB, Fleischer DE et al. Results from the American Society for Gastrointestinal Endoscopy/U.S. Food and Drug Administration collaborative study on complication rates and drug use during gastrointestinal endoscopy. Gastrointest Endosc 1991; 37: $421-427$

73 Chan MF. Complications of upper gastrointestinal endoscopy. Gastrointest Endosc Clin N Am 1996; 6: 287- 303

74 Hart R, Classen M. Complications of diagnostic gastrointestinal endoscopy. Endoscopy 1990; 22: 229-233

75 Hernandez LV, Jacobson JW, Harris MS. Comparison among the perforation rates of Maloney, balloon, and savary dilation of esophageal strictures. Gastrointest Endosc 2000; 51: 460-462

76 Kavic SM, Basson MD. Complications of endoscopy. Am J Surg 2001; 181: 319-332

77 Iannettoni MD, Vlessis AA, Whyte RI et al. Functional outcome after surgical treatment of esophageal perforation. Ann Thorac Surg 1997; 64: 1606 - 1609; discussion 1609-1610

78 Montgomery E, Bronner MP, Goldblum JR et al. Reproducibility of the diagnosis of dysplasia in Barrett esophagus: a reaffirmation. Hum Pathol 2001; 32: $368-378$

79 Ormsby AH, Petras RE, Henricks WH et al. Observer variation in the diagnosis of superficial oesophageal adenocarcinoma. Gut 2002; 51: 671-676

80 Provenzale D, Kemp JA, Arora $S$ et al. A guide for surveillance of patients with Barrett's esophagus. Am J Gastroenterol 1994; 89: 670-680

81 Provenzale D, Schmitt $C$, Wong JB. Barrett's esophagus: a new look at surveillance based on emerging estimates of cancer risk. Am J Gastroenterol 1999; 94: 2043-2053

82 Alikhan M, Rex D, Khan A et al. Variable pathologic interpretation of columnar lined esophagus by general pathologists in community practice. Gastrointest Endosc 1999; 50: 23-26

83 Zaman A, Hapke R, Sahagun G et al. Unsedated peroral endoscopy with a video ultrathin endoscope: patient acceptance, tolerance, and diagnostic accuracy. Am J Gastroenterol 1998; 93: 1260-1263

84 de Boer AG, Stalmeier PF, Sprangers MA et al. Transhiatal vs extended transthoracic resection in oesophageal carcinoma: patients' utilities and treatment preferences. Br J Cancer 2002; 86: 851-857

85 Weinstein MC, Siegel JE, Gold MR et al. Recommendations of the Panel on Cost-effectiveness in Health and Medicine. JAMA 1996; 276: $1253-1258$ 

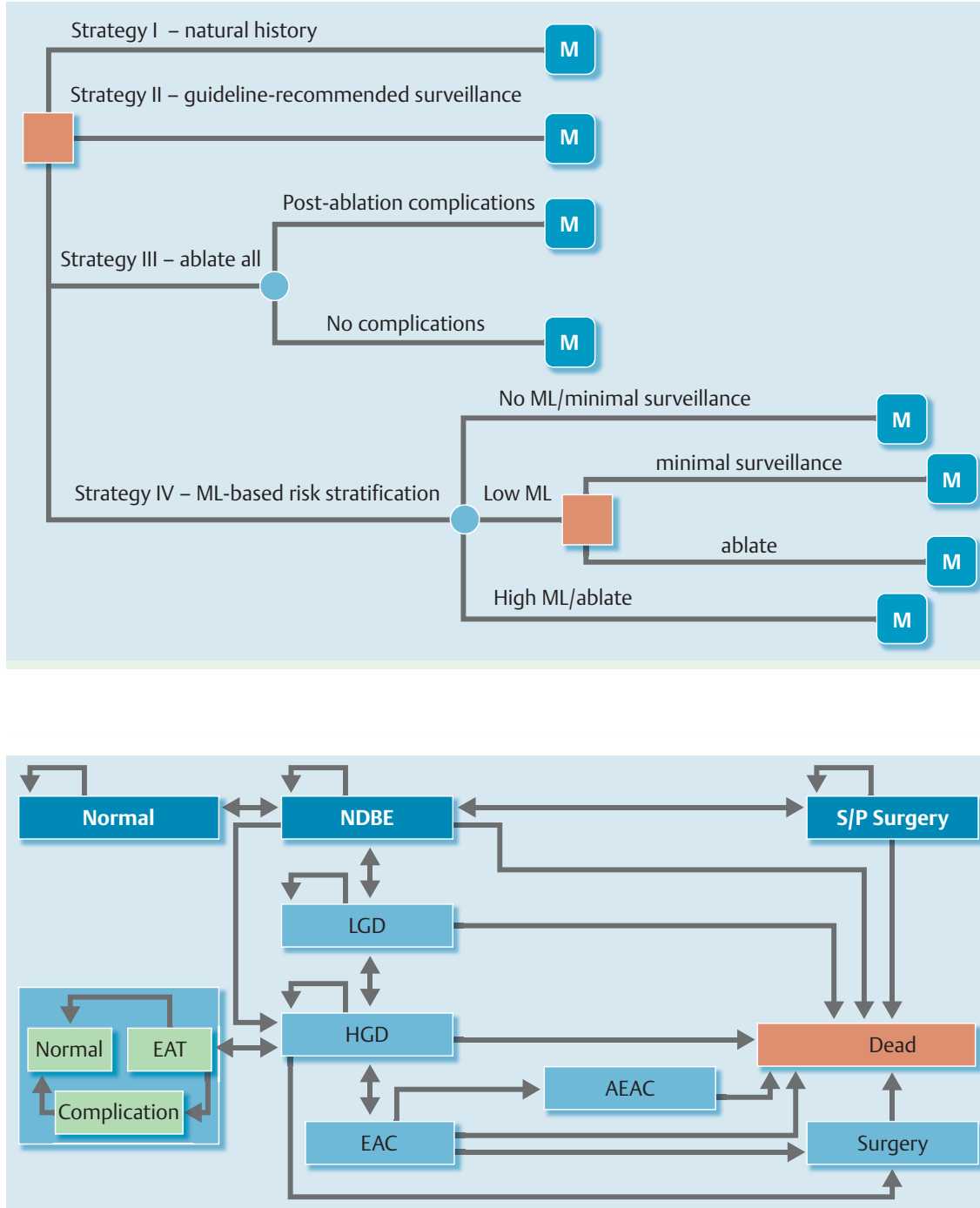

Supplementary Fig. 1 The hybrid model of the linear decision tree terminating in Markov models. In the decision tree, a square node represents the decision node at entry, the filled circles are chance nodes, and the circles with the letter $\mathrm{M}$ represent Markov nodes. In strategy I, after the diagnosis of nondysplastic Barrett's esophagus (NDBE) was established, the natural history of this condition was modeled without any specific intervention. In strategy II, all patients with NDBE underwent periodic endoscopic surveillance according to the current guidelines of the American College of Gastroenterology. In strategy III, all patients with NDBE underwent endoscopic ablation. In strategy IV, all patients with NDBE underwent risk stratification for esophageal adenocarcinoma, and subsequently ablation, based on mutational load (ML); Patients with no ML underwent minimal surveillance, patients with low ML underwent standard surveillance or ablative therapy (another decision point), and patients with high ML were treated selectively with endoscopic ablation.
Supplementary Fig. 2 Health and disease state transitions in the Markov model. The natural history of patients with nondysplastic Barrett's esophagus (NDBE) was modeled for various health and disease states, each associated with a different set of costs and utilities. A single arrowhead indicates transition from one state to another in the direction of the arrowhead; double arrowheads indicate that transitions in both directions are allowed in the model. Half-circle arrowheads represent states in which a patient can remain indefinitely. The light blue area represents stages associated with endoscopic ablative therapy (EAT), if allowed in the model. Normal, no Barrett's esophagus; LGD, low grade dysplasia; HGD, high grade dysplasia; EAC, esophageal adenocarcinoma; AEAC, advanced esophageal adenocarcinoma; S/P Surgery, status post surgery.

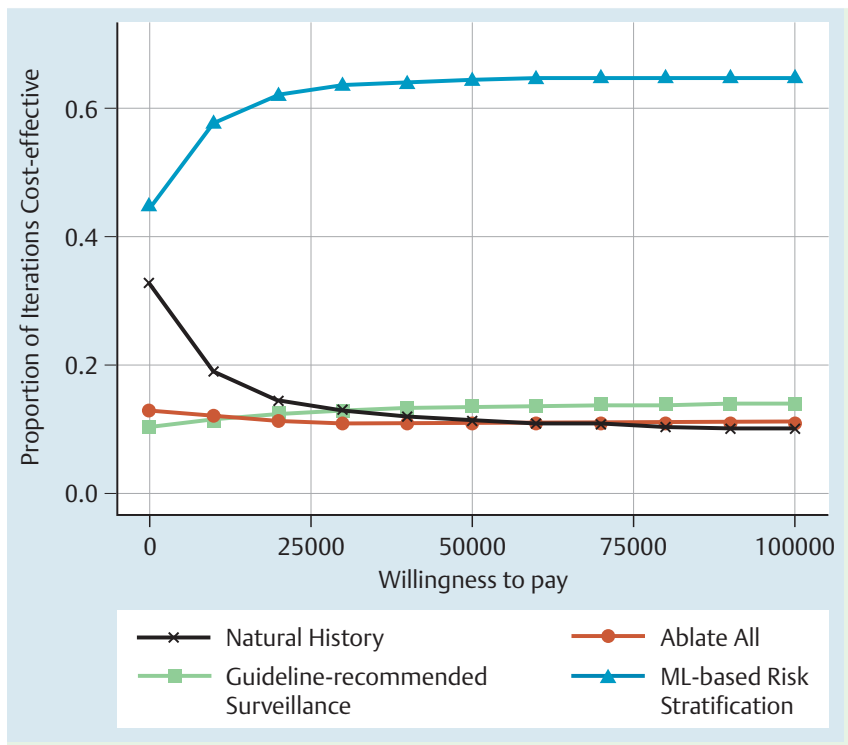

Supplementary Fig. 3 The proportions of iterations in the Monte Carlo analysis ( $y$-axis) that are acceptable as cost-effective for each strategy against increasing willingness to pay (x-axis). A strategy of risk stratification based on mutational load (strategy IV, blue) was the most cost-effective strategy in terms of incremental net health benefit, particularly when compared with the currently practiced strategy of endoscopic surveillance (strategy II, green). 
Origina a ticle

\begin{tabular}{|c|c|c|c|}
\hline Input variable & Reference case estimate & Range & References \\
\hline \multicolumn{4}{|l|}{ Annual rate of progression in an average cohort of patients with NDBE, \% } \\
\hline HGD to cancer & 0.07 & $0.001-0.3$ & {$[35,46-48]$} \\
\hline NDBE to HGD & 0.01 & $\begin{array}{l}0.0028- \\
0.083\end{array}$ & {$[35,46,49,50]$} \\
\hline NDBE to cancer & 0.005 & $0.001-0.1$ & {$[35,51-55]$} \\
\hline NDBE to LGD & 0.05 & $0.01-0.078$ & $\begin{array}{l}{[35,47,49,53,56,} \\
57]\end{array}$ \\
\hline LGD to HGD & 0.05 & $0.001-0.078$ & {$[35,36,47,50,56]$} \\
\hline LGD to cancer & 0.01 & $0.005-0.05$ & [36] \\
\hline Risk factor expressed as multiple of annual rate of progression in patients with & & & {$[27-31]$} \\
\hline No ML & 0.1 & $0.05-1$ & \\
\hline Low ML & 1 & $0.5-5$ & \\
\hline High ML & 2.5 & $1-10$ & \\
\hline Prevalence of ML in patients with NDBE, \% & & & {$[27,28]$} \\
\hline Low ML & 30 & $5-75$ & \\
\hline High ML & 8 & $1-25$ & \\
\hline \multicolumn{4}{|l|}{ Annual rate of regression, $\%$} \\
\hline HGD to NDBE & 0.1 & $0.01-0.15$ & {$[35,47,50]$} \\
\hline HGD to LGD & 0.07 & $0.05-0.1$ & {$[35,46-50]$} \\
\hline NDBE to normal & 0.0175 & $0.001-0.02$ & {$[35,58,59]$} \\
\hline \multicolumn{4}{|l|}{ Efficacy of surgical treatment } \\
\hline Probability of surgical resectability & & & {$[35,46,60-64]$} \\
\hline Diagnosis by surveillance & 0.8 & $0.5-1.0$ & \\
\hline Diagnosis by symptoms & 0.5 & $0.1-0.7$ & \\
\hline \multicolumn{4}{|l|}{ Probability of curative resection } \\
\hline Diagnosis by surveillance & 0.7 & $0.6-0.9$ & \\
\hline Diagnosis by symptoms & 0.2 & $0.1-0.43$ & \\
\hline \multicolumn{4}{|l|}{ Efficacy of ablative therapy } \\
\hline Probability of complete ablation after three sittings & 0.5 & $0.1-1.0$ & {$[35,38,65,66]$} \\
\hline Probability of complications with ablation & & & {$[35,38,65,66]$} \\
\hline Total & 0.1 & $0-0.25$ & \\
\hline Perforation & 0.05 & $0-0.15$ & \\
\hline Stricture & 0.05 & $0-0.15$ & \\
\hline \multicolumn{4}{|l|}{ Mortality, \% } \\
\hline From esophagectomy & 0.04 & $0.02-0.20$ & {$[67-71]$} \\
\hline From endoscopy & 0.00002 & $0-0.00005$ & {$[72-76]$} \\
\hline From surgery for repair of esophageal perforation & 0.08 & $0.05-0.15$ & [77] \\
\hline From advanced esophageal adenocarcinoma (annual probability) & 0.6 & $0.3-1.0$ & [34], Assumption \\
\hline \multicolumn{4}{|l|}{ Misdiagnosis rates } \\
\hline Cancer called HGD & 0.175 & $0.01-0.0 .2$ & {$[35,78-81]$} \\
\hline Cancer called LGD & 0.05 & $0.01-0.10$ & {$[35,78-81]$} \\
\hline HGD called cancer & 0.11 & $0.01-0.20$ & {$[35,78-81]$} \\
\hline HGD called LGD & 0.115 & $0.01-0.20$ & {$[35,78-81]$} \\
\hline LGD called cancer & 0.05 & $0.01-0.10$ & {$[35,80,81]$} \\
\hline LGD called HGD & 0.083 & $0.01-0.10$ & {$[35,80-82]$} \\
\hline NDBE called HGD & 0.01 & $0.0-0.02$ & {$[32,81]$} \\
\hline NDBE called normal & 0.01 & $0.0-0.0 .01$ & {$[32,81,83]$} \\
\hline Normal called NDBE & 0.01 & $0.0-0.005$ & [34] \\
\hline \multicolumn{4}{|l|}{ Patient preferences (utilities) for health states } \\
\hline NDBE & 1 & $0.8-1.0$ & {$[35,81]$} \\
\hline LGD/HGD & 0.99 & $0.8-1.0$ & [37] \\
\hline After esophagectomy & 0.97 & $0.8-1.0$ & {$[34,36,37,81,84]$} \\
\hline Early cancer & 0.9 & $0.8-1.0$ & [81] \\
\hline Late cancer & 0.34 & $0-1$ & {$[81,84]$} \\
\hline Stricture related to ablative therapy & 0.97 & $0.85-1.0$ & [81] \\
\hline
\end{tabular}

HGD, high grade dysplasia; NDBE, nondysplastic Barrett's esophagus; LGD, low grade dysplasia; ML, mutational load. 
Supplemental Table 2 Estimates of key costs for the management of nondysplastic Barrett's esophagus.

\begin{tabular}{|llll}
\hline Aspect of management & Reference case cost, US\$ & Range, US\$ & References \\
\hline Esophagectomy & 19,000 & $10,000-40,000$ & {$[34,35,37,80]$} \\
\hline EGD with ablation & 10,000 & $5000-25,000$ & {$[37]$, Assumption } \\
\hline EGD with biopsies & 830 & $350-1200$ & {$[34,35]$} \\
\hline Treatment for ablation-related stricture & 2500 & $1000-3000$ & {$[35,80]$} \\
\hline Chemotherapy & 10,750 & $\pm 25 \%$ & {$[32,37]$} \\
\hline Radiation & 5400 & $\pm 25 \%$ & {$[32,37]$} \\
\hline Annual follow-up after surgery for early cancer (first 5 years) & 1500 & $400-2000$ & {$[37]$} \\
\hline Hospice & 6228 & $0-7$ & {$[37]$} \\
\hline Discount rate, \% & 3 & $2500-7500$ & CMS
\end{tabular}

US, United States; EGD, esophagogastroduodenoscopy; ML, mutational load; CMS, Centers for Medicare \& Medicaid Services. 